\title{
El Carnaval de los cuerpos. El desafío simbólico y político del trans-vestismo*
}

\author{
Carnival of the bodies. \\ The symbolic and political challenge of Transvestitism
}

LIBERA PISANO**

\begin{abstract}
Resumen: Esta contribución investiga el travestismo, entendido como una constante cultural y como un desafío simbólico y político a las indentidad del género. A partir de las reflexiones de Judith Butler, el travestismo será analizado de acuerdo con tres aspectos: una inversion estética, un acto performativo y una subversión semántica. Palabras clave: Trans-vestismo, Drag, Género, Cuerpo, Performativo, Butler.
\end{abstract}

\begin{abstract}
This paper discusses cross-dressing as a cross-cultural phenomenon and as a symbolic and political challenge to gender's identity as well. Starting from Judith Butler's works, the drag will be analyzed in accordance with three different aspects: an aesthetic inversion, a performative act and a semantic turn.

Keywords: Transvestitism, Drag, Gender, Body, Performativity, Butler.
\end{abstract}

En esta contribución pretendo investigar el travestismo, entendido como práctica semiótica y como dispositivo de deconstrucción de la identidad, señalando cómo la puesta en escena del sí a través del cross-dressing representa un desafío simbólico y político que posibilita leer el cuerpo como un territorio abierto de significados. No voy a ocuparme de transexualismo, sino de travestismo -desde un enfoque teorético- como inversión y puesta en escena constante de la representación del otro. No se trata solamente de las performance de las drag queens o de los drag kings, sino de un pliegue del género, de una práctica de

Fecha de recepción: 29/05/2016. Fecha de aceptación: 17/07/2016.

* Traducción de Cristina Basili

** Libera Pisano es Junior Fellow en el Maimonides Centre for Advanced Studies de la Universidad de Hamburgo. Su investigación se centra en la filosofía del lenguaje, en los estudios judíos, en la tradición anarquista y utopíca, en los estudios de género. Entre sus últimas publicaciones: Lo spirito manifesto. Percorsi linguistici nella filosofia hegeliana (ETS, Pisa 2016); Misunderstanding Metaphors. Linguistic skepticism in Mauthner's philosophy, Yearbook Maimonides Centre for Advanced Studies (De Gruyter, Berlin-New York 2016); Anarchia dello spirito - spirito dell'anarchia. Su Gustav Landauer, in "Il Cannocchiale", XXXVIII, 3, Autumn 2013, pp. 163-170. En 2015 editó el número de "Azimuth. Philosophical coordinates in modern and contemporary Age" con el titulo Performing the obscenity. Bodies, power and desire in the contemporary debate. Email: libpisano@gmail.com 
gender blending ${ }^{1}$. Aterrador y seductor, el travesti encarna el elemento de discontinuidad, el monstrum que se escapa a cualquier posibilidad de definición. En efecto, no es casualidad que se haya tolerado solamente en el ámbito teatral y que el único lugar en el que la transgresión estaba permitida haya sido el perímetro del palco escénico. Sin embargo, el travestismo, como cada elemento fronterizo y cada sexualidad periférica ${ }^{2}$, compromete la definición de la identidad de género y desvela el criterio de control y de demarcación que subyace al estereotipo inscrito en el cuerpo. Desmantelar estos criterios de control e identificación constituye siempre una operación política, por esta razón el travestismo lanza un desafío perturbador que compromete el espacio de definición de la identidad desde su interior.

Definir el cross-dressing no es tarea fácil, puesto que -aunque se trate de un fenómeno marginal y estigmatizado, en muchas ocasiones, como una desviación ${ }^{3}$ - sigue siendo una constante cultural y antropológica que puede encontrarse en diversas épocas. El tema no se ha investigado mucho porque siempre se ha considerado como un fenómeno individual, aunque tenga que ver, en realidad, con un tabú cultural y con procesos de remoción colectiva. Aunque no pueda separarse de la elección del objeto sexual, la primera aparición del término transvestite se remonta a Magnus Hirschfeld, un pionero de los estudios de género, que no habla de ello como de un signo de distinción de la homosexualidad 4 .

El travestismo ha existido siempre y su historia es larga ${ }^{5}$. Gracias a los testimonios de fuentes escritas e iconográficas, sabemos que los travestis han sido sacralizados o catalogados como excentricidades de la naturaleza, estigmatizados o mitificados. En el mundo antiguo fueron muchos los episodios de camuflaje engañoso o de travestismos rituales, pero se trataba de prácticas admitidas en los confines de lo humano y de perturbadoras experiencias límites ${ }^{6}$. No es por casualidad que -dejando de lado a los dioses y de los héroes de la

1 Cf. R. Ekins y D. King (eds.), Blending genders. Social aspects of cross-dressing and sex-changing, Routledge, New York, 1996.

2 La expresión pertenece a Foucault. Cf. Foucault, M., La volonté de savoir, Gallimard, Paris, 1976.

3 Sobre el travestismo como patología, cf. Storr, A., «The Psychopathology of Fetischism and Transvestitism», en: A. Samuels (eds.): Psychopathology: contemporary Jungian perspectives, The Guilford Press, New York, 1991, pp. 255-274.

4 Cf. Hirschfeld, M., Die Transvestiten: Eine Untersuchung über den erotischen Verkleidungstrieb, mit umfangreichem kasuistischem und historischem Material, Verlag Alfred Pulvermacher, Berlin, 1910.

5 Frazer en The golden bough cita muchos ejemplos de cross-dressing, refiriendo múltiples casos de travestis que tienen un estatuto especial en la historia y en la etnografía: desde los berdaches de los nativos americanos hasta los chamanes siberianos, desde los travestis brasileños hasta los bantut de las islas Filipinas, desde los xanith del Omán hasta los hijras de la India. Cf. Frazer, J., The golden bough. A study in magic and religion, Macmillan, New York, 1915. Siguiendo a Frazer, Crawley nos ha propiciado uno de los estudios más completos acerca del cross-dressing y de los antiguos ritos de iniciación. Cf. Crawley, A.E., «Achilles at Skyros», The Classical Review, n 6, Juno 1893, pp. 243-45; Id., «Sexual Taboo: a study in the relations of the sexes», Journal of the Anthropological Institute, ${ }^{\circ} 24$, Juno 1895, pp. 116-25.

6 En la Antigüedad la construcción del género no estaba separada de la narración religiosa, en particular de la representación retórica y de las maneras de argumentarla. En Grecia, según el cuerpo hipocrático, existían tres categorías: hombres, mujeres y andróginos o andreiai (mujeres con rasgos masculinos). El cuerpo contenía elementos masculinos y femeninos, definidos por un número exacto de reglas y actos (esta idea se encuentra también en Filón de Alejandría, según el cual lo masculino y lo femenino eran categorías filosóficas). La mitología griega consta de múltiples episodios de cross-dressing: el caso de Hércules es emblemático; representa tanto el hombre viril cuanto -debido a la dualidad que lo marca desde su nacimiento (Zeus y Alcmena) - un hombre con una sexualidad polivalente y compleja, que se hace visible en las estatuas y las pinturas que le retraen (baste con pensar en el episodio en el que ama al joven Hylas o el episodio de los disfraces con la reina Ónfale, que 
mitología- solo los sacerdotes, los cómicos y, en la Roma antigua, las prostitutas hayan sido las únicas categorías en las que se permitía el travestismo. Si dejamos de un lado el travestismo medieval y el travestismo barroco, que merecerían un estudio más exhaustivo, en el siglo XIX asistimos a una progresiva medicalización del fenómeno, puesto que la sexualidad empieza a configurarse como disciplina aparte ${ }^{7}$. En el siglo XX -desde los años sesenta en adelante-, el travestismo se ha transformado en un territorio de experimentación de nuevas formas de subjetividad, en un desafío simbólico a las autoridades y a los modelos dominantes, lo que hace que se haya percibido como una amenaza. En las últimas décadas, cuando el cuerpo se ha vuelto un trampolín para la filosofía, el travestismo ha sido objeto de estudios, en cuanto dispositivo teórico-práctico que pone en crisis la univocidad de los significados de la heteronormatividad ${ }^{8}$. En este caso, se habla del desafío que el queer lanza al género y al esquema binario de la heterosexualidad. Existen por lo menos tres modalidades a través de las cuales el travestismo cuestiona el estatuto de nuestro cuerpo, formulando preguntas importantes desde el punto de vista teorético. Podemos distinguir un aspecto estético, uno performativo y otro semántico.

\section{Estético}

El cross-dressing manifiesta una disonancia estética y cultural que, sin embargo, está relacionada a la necesidad antropológica de los hombres de cambiar su propio cuerpo ${ }^{9}$. El vestuario desempeña en este caso un papel central, porque representa un Gestalt que engloba

lo obligó a disfrazarse de mujer y a filar la lana). Sin embargo, Hércules no representa un caso aislado. Tiresias había sido mujer durante una parte de su vida, Aquiles se disfraza de mujer para encontrar a Ulises y cabría mencionar a Dioniso, Apolo, Diana, las Amazonas, etc. Para los héroes de la mitología disfrazarse de mujer no era un desafío al género, sino una experiencia límite que estaba prohibida a los hombres. Por lo que concierne la Roma antigua, la estructura retórica sobre la que se ha construido el Imperio durante la época de Augusto era la de una política de la masculinidad: la élite romana se había redefinido a sí misma en oposición a la feminidad de los griegos; el vir era el hombre en contraposición a la androginia de los griegos. No es por casualidad que los romanos utilizaban la palabra griega tribas para referirse al monstrum-gender que constituía una poderosa herramienta de propaganda retórica de protección respecto de una posible perdida de virilidad y, asimismo, permitía evitar que en la figura de la matrona -ya muy masculinizada- se produjera una desviación de género. Cf. C.J. Summers (eds.), The queer Encyclopedia of the visual arts, Cleis, San Francisco, 2004; J.P. Hallett y M.B. Skinner (eds.), Roman sexualities, University Press, Princeton, 1997; T. Penner y C. Vander Stichele, Mapping gender in ancient religious discourses, Brill, Leiden-Boston, 2007; F.I. Zeitlin, J.J. Winker \& D.M. Halperin (eds.), Before sexuality. The construction of erotic experience in the ancient Greek world, University Press, Princeton, 1991.

7 Cf. Foucault, M., Historie de la sexualité, Gallimard, Paris, 1976.

8 Sobre este tema Marjorie Garber ha escrito un libro crucial: Vested interests. Cross-dressing and cultural anxiety, Routledge, New York, 1992. La autora examina el travestismo en muchas de sus formas, desde Shakespeare hasta Twain, desde Peter Pan hasta David Bowie y subraya cómo el carácter de tercero excluido, ni masculino ni femenino, es el punto de partida de la invención producida por la cultura, es decir, de la puesta en cuestión del esquema binario del pensamiento occidental. El travesti es, de hecho, lo que no tiene afiliación, lo que puede sobreponerse a la historia de la homosexualidad solo de forma contingente. Según Garber sin travestismo no puede haber cultura porque el travestismo es la incesante superación del esquema binario, lo que lleva hacia lo nuevo. Cf. también Newton, E., Mother Camp. Female Impersonators in America, Chicago, 1972; Ekins, R., Male femaling. A grounded theory approach to cross-dressing and sex-changing, Routledge, New York, 1997.

9 Cf. R. Barnes y J.B. Eicher (eds.), Dress and gender making and meaning, Berg, Oxford, 1992. 
el cuerpo en todas sus manifestaciones, un signo inequívoco que incluye y excluye al mismo tiempo, pero sobre todo un indicador y un productor de género. El vestido tiene valor de medium y de artefacto, juega con la identidad y la imagen de sí, según un criterio tangible de estructuras normativas ${ }^{10}$. El traje es el pasaporte que permite el tránsito hacia lo prohibido porque funciona como una marca de sentido. Por esta razón, el travestismo es un desplazamiento revelador que amenaza la iconografía de género. Imitando el género opuesto, el drag corrobora y, al mismo tiempo, toma las distancias desde los estereotipos, pone y quita el significado ${ }^{11}$. En la raíz de todo esto se encuentra la rebelión iconoclasta contra los confines tradicionales de género. Un hombre disfrazado de mujer, o al revés una mujer disfrazada de hombre, por trámite de la inversión del vestuario, supone un desafío a unas categorías de lo masculino y de lo femenino consideradas esenciales, biológicas o culturales. Este desafío empieza por una estética de la existencia: la figura del travesti ejerce, como ninguna otra, un extraordinario poder de desquiciamiento estético.

\section{Performativo}

El segundo aspecto está conectado, de forma directa, con la noción de performativo en relación al género, a partir de las reflexiones de Judith Butler. En su obra Gender Trouble la filósofa desnaturaliza el género que, de esta manera, se presenta como una performance y un ámbito de acción que puede ser deconstruido y contestado ${ }^{12}$. Lo esencial, en la reflexión de Butler, es el cambio desde una concepción sustancial de la identidad a un género entendido como un campo sedimentado de discursos, prácticas y estereotipos, abierto a posibilidades de reescritura. Asumir la performatividad del género significa tomar distancias del dualismo metafísico de alma y cuerpo, resistir a la violencia normativa y desarraigar la concepción de la heterosexualidad como algo natural. El género como gesto elimina la idea de esencia interior y se revela como el resultado de una repetición ritual de actos y significados ya establecidos y por eso legitimados. Una repetición tal crea la ilusión de que el género es algo natural, una

10 El discurso acerca de la moda abre una paréntesis muy interesante de la que no podemos ocuparnos aquí: desde Simmel hasta Benjamin que la define como el sex-appeal de lo inorgánico hasta Barthes, para el cual se produciría un desplazamiento a una teoría de la moda entendida como discurso social: la ropa es un lenguaje y el cuerpo vestido es un territorio físico-cultural en el que se realiza la performance. Un paralelo entre moda y lenguaje había sido esbozado en el Cours de linguistique generale de Saussure, que subraya cómo la moda, aunque de forma arbitraria, no puede alejarse en definitiva de las condiciones establecidas por el cuerpo; además considera la imitación el motor fundamental tanto de los cambios fonéticos cuanto de la moda. Cf. C. Baldini (eds.), Sociologia della moda, Armando, Roma, 2008.

11 Sobre la corroboración del esquema binario en el drag, cf. Garber, M., Vested interest, cit., p. 109: «The phenomenon of transsexualism is both a confirmation of the contractedness of gender and a secondary recourse to essentialism - or to put it a slightly different way, transsexualism demonstrates that essentialism is cultural construction». Por esta misma razón, la crítica feminista no siempre ha estado de acuerdo con el $d r a g$, entendido como una forma de low camp, porque propone algunos estereotipos misóginos de lo femenino. Para mencionar solo algunos de los artículos sobre la interpretación del cross-dressing en la literatura feminista, cf. R.M. Dekker y L. van del Pol (eds.), The tradition of female transvestitism in the early modern Europe, Macmillan Press, Basingstoke, 1989; H. Devor, FTM: Female-to-Male Transsexuals in Society, Indiana, Indiana University Press, 1999; J. Wheelwright, Amazons and military maids: women who dressed as men in the pursuit of life, liberty and happiness, Pandora Press, London, 1989; J. Epstein y K. Straub (eds.), Bodyguards: the cultural politics of gender ambiguity, Routledge, New York, 1991.

12 Butler, J., Gender Trouble, Routledge, New York, 1990. 
sustancia constante. Sin embargo, el drag permite desvelar este dispositivo. Los actos a través de los cuales se estructura el género intentan acercarse al fundamento sustancial de la identidad, pero en realidad justo en el momento en que este mecanismo es discontinuo y deja de funcionar -como en el caso del $d r a g$ - el fundamento se revela contingente y temporal. Para Butler el drag, imitando el género, revela de forma implícita la estructura imitativa del género mismo y su contingencia. De esta manera aflora la gracilidad de la realidad del género con su parodia de la noción de verdadera identidad. La vitalidad subversiva y transformativa del drag desplaza el paradigma de la heterosexualidad y le da la vuelta al régimen discursivo dominante, reflejando la imitación por la cual se reproduce el género. Gracias al travestismo el género se revela como un a posteriori, una serie de actos estilizados repetidos en un cuadro rígido de reglamentaciones establecidas en el tiempo, que produce la ilusión de la sustancia natural. El desenmascaramiento revela la violencia consustancial a los estereotipos de género. Las normas establecen el "nosotros" por trámite de un ejercicio del poder que incluye y excluye y sancionan el ámbito en el que se puede conceder legitimidad a una determinada clase de cuerpos. Todos los demás cuerpos, los que no corresponden a estos parámetros, son considerados falsos, irreales, ilegítimos y constituyen precisamente el ámbito de de-humanización y abyección respeto al cual se constituye lo humano. En el drag, la falta de correspondencia entre sexo biológico, gender identity y gender performance produce una discrepancia que desnaturaliza la equivalencia usual. A través de una práctica paródica y mimética, cabe desmontar la lógica excluyente de la normatividad heterosexual y desestabilizar su régimen de significación. Sin embargo, en la introducción a la segunda edición de Gender Trouble, Butler declara que la discusión del drag contenida en el libro no ha de ser entendida como un ejemplo de subversión política, sino como aquello que pone en discusión la realidad del género ${ }^{13}$. En el travestismo, el género aparece como una realidad ilusoria, como apariencia, artificio, juego y se ponen en duda las categorías con las cuales codificamos y leemos los cuerpos en su principal diferencia. Esta duda no permite distinguir entre lo real y lo irreal de modo que la idea de realidad de género emerge como una utopía. Sin embargo, el espectro detrás de la identidad es el del sujeto, una categoría central para la política, que procede según determinados objetivos de inclusión y de exclusión, escondidos detrás de una sospechosa naturalización. En sus obras sucesivas, Butler desarrolla esta dimensión política en la búsqueda de una ética de la vulnerabilidad como rasgo distintivo de la condición humana ${ }^{14}$.

\section{Semántico}

El último aspecto está relacionado con la deconstrucción y la construcción del significado que conlleva el travestismo. Aceptar el género - tal como propone Teresa De Laurentis (1996) releyendo a Foucault- como «un processo di una serie di tecnologie sociali» ${ }^{15}$ significa leer el cuerpo como un acto discursivo. El cuerpo no es una superficie pasiva a la espera de significación, sino un campo político cuyos confines resultan siempre negociables. Lo masculino y lo femenino se construyen a través de performances sociales y prácticas de significación. También

13 Butler, J., Gender Trouble, 2 ed., Routledge, New York, 2006.

14 Cf. Butler, J., Bodies that matter, Routledge, New York, 1993; Ead., Undoing Gender, Routledge, New York, 2004.

15 De Lauretis, T., Sui generis. Scritti di teoria femminista, Feltrinelli, Milano, 1996, p. 132. 
la identidad es un significado que hay que negociar, una práctica y un efecto de discursos que no tienen carácter absoluto. De hecho, el sujeto no se determina de una vez para siempre; la significación que lo constituye es un proceso escondido reglamentado por repeticiones que tienen efectos sustanciales. Aceptar la subversión de esta gramática significa descolocar las normas del género y hacer del cuerpo el palco escénico de una performance disonante, que revela la misma performance. El género está abierto: es un acto de la auto-parodia de lo natural y de lo original. El reconocimiento de esta contingencia radical es el vértigo de la performance, porque se trata de una parodia que no tiene original; es la parodia de la misma noción del original, una apertura hacia la re-significación y la desnaturalización del significado. Como el mundo al revés de Hegel, el drag desquicia la distinción entre natural y artificial, entre interno y externo, entre profundidad y superficie. Propio/impropio, interno/externo, masculino/femenino, construcción/esencia: todos términos de un dualismo sin síntesis. Sin embargo, no se trata de un dualismo trágico, sino paródico, casi carnavalesco en el sentido bajtiniano del término. Me refiero al realismo grotesco que está a la base del carnaval, cuyo aspecto principal es la enorme importancia que se atribuye al cuerpo, entendido no como cuerpo individual, aislado y privado, sino como cuerpo que pertenece al pueblo, cuerpo infinito: «en el realismo grotesco [...] el principio material y corporal aparece bajo la forma universal de fiesta utópica ${ }^{16}$. El cuerpo grotesco es, así pues, un cuerpo abierto hacia el mundo y la estética del grotesco es una estética del devenir en la cual el cuerpo tiene confines establecidos e indeterminados y queda lejos de una concepción clásica, acabada, cerrada y perfecta.

Teniendo en cuenta estas premisas, cabe admitir la existencia de una hermenéutica del travestismo, una relación dual entre el otro y el travesti, entre quien lee y quien está leído, una técnica del «double reading gender» ${ }^{17}$. Este otro, en realidad, no representa únicamente algo exterior, sino que comparte el mismo cuerpo del travesti que aparece, de este modo, como el campo de batalla entre lo masculino y lo femenino, el lugar de una repulsión, de una degeneración y de una (de)construcción siempre precaria del sí. Además, justamente porque -como afirma Mary Douglas- la piel está recorrida por el tabú y por transgresiones anteriores, el cuerpo representa una sinécdoque del sistema social, un margen peligroso en donde confluyen aperturas y contaminaciones, un confín variable ${ }^{18}$.

16 Bajtin, M., La cultura popular en la edad media y en el renacimiento (1965), trad. cast. de J. Forcat y C. Conroy, Alianza, Madrid, 2003, p. 19. Como es sabido, Bajtin considera el carnaval como la expresión más clara, entre todos los fenómenos colectivos, de la comicidad popular de la Edad media; una percepción cómica de la realidad opuesta a la concepción seria de las cosas. En la modernidad con la institución de las jeraquías sociales se habría consolidado una concepión mutilada de la risa, idiferente en relación al aspecto positivo de regeneración presente en la Edad media. La risa carnavalesca autentica, en efecto, representa la muerte y la vida, una risa colectiva que tiene como su objetivo polémico el orden existente. En el carnaval no hay palcos escénicos, ni actores; es la vida misma que se espectaculariza a través del juego en el que se combinan lo sacro y lo profano, el alto y lo bajo, el rico y el pobre, el hombre y la mujer. A través de la risa y de la parodia se reduce al nivel corpóreo todo aquello que es espiritual. Sin embargo, este gesto de rebajar es igual a llevar hacia la tierra, hacia la muerte y el vientre. A partir del siglo XVII se impone una estatalización de la vida de la fiesta, que se transforma en un desfile, los ritos degeneran y el imaginario comico carnavalesco empieza a definirse a través de una serie de críterios formales para luego confluir en la novela moderna donde pierde su carácter de subversión.

17 Garber, M., Vested interests, cit., p. 168.

18 Cf. Douglas, M., Purity and Danger. An Analysis of Concepts of Pollution and Taboo (1966), Routledge, New York, 2002. 
Parafraseando lo que Humboldt ha afirmado acerca del lenguaje, podemos decir que el género no es algo dado, sino un devenir, no es ergon, sino energeia ${ }^{19}$. El cross-dressing representa una excepción que implica una rotura de las reglas del lenguaje. Podríamos decir, con Barthes, que el cuerpo es un sistema retórico de significación y la disfunción -el drag en nuestro caso- de un sistema unívoco de significados implica uno shift permanente ${ }^{20}$. La gramática, una de las herramientas más poderosas para establecer la identidad de género, viene alterada a partir del cuerpo y tal excentricidad pone en discusión la obviedad de la identificación. Sobre el cuerpo está en marcha una ley de la cita de la cita y de la repetición de la repetición ${ }^{21}$. En su exhibición hiperbólica del artificio el drag representa un exceso de significado, descoloca la puntuación y la gramática del cuerpo. En la fiesta de los cuerpos, entendida como suspensión de la norma, del lenguaje y del tiempo ordinario y en la puesta en escena paródica del drag, se esconde el pliegue del género, un fenómeno perturbador que señala en la idea de un cuerpo abierto, expuesto a una re-significación anárquica sin original, siempre y de nuevo posible, un antídoto contra la violencia de la norma.

\section{Bibliografia}

Bajtin, Mijail (1965, 2003): La cultura popular en la edad media y en el renacimiento, trad. cast. de J. Forcat y C. Conroy, Alianza, Madrid.

Baldini, Costanza (eds.) (2008): Sociologia della moda, Armando, Roma.

Barnes, Ruth y Eicher, Joanne B. (eds.) (1992): Dress and gender making and meaning, Berg, Oxford.

Barthes, Roland (1967): Système de la mode, Seuil, Paris.

Butler, Judith (1993): Bodies that matter, Routledge, New York.

Butler, Judith (2004): Undoing Gender, Routledge, New York.

Butler, Judith (2006): Gender Trouble, 2 ed., Routledge, New York.

Crawley, Alfred E. (Juno 1893): «Achilles at Skyros», The Classical Review, n 6, pp. 243-45.

Crawley, Alfred E. (Juno 1895): «Sexual Taboo: a study in the relations of the sexes», Journal of the Anthropological Institute, $\mathrm{n}^{\circ} 24$, pp. 116-25.

De Lauretis, Teresa (1996): Sui generis. Scritti di teoria femminista, Feltrinelli, Milano:

Dekker, Rudolf M. y Van Del Pol, Lotte (eds.) (1989): The tradition of female transvestitism in the early modern Europe, Macmillan Press, Basingstoke.

19 Cf. Von Humboldt, W., Über die Verschiedenheit des menschliches Sprachbaues, Akademie, Berlin, 1836.

20 Cf. Barthes, R., Système de la mode, Seuil, Paris, 1967.

21 Acerca del exceso de significado y de la práctica de la cita, no está de sobra apuntar a la distinción entre camp y drag. Las Notas sobre lo "camp" de Susan Sontag - aunque muy controvertidas - representan el primer documento que ofrece una definición del camp. Si el camp representa una práctica de la cita, el drag vehicula un exceso de sentido y presenta un desafío al cuerpo cerrado. En ambos casos está presente la exhibición de lo perturbador, porque se muestra una dualidad que no tiene conciliación, un exceso de significado (baste con pensar, por ejemplo, en que el camp actualiza objetos de épocas pasadas para mostrar su exceso de significación). Ambos ofrecen una versión bufonesca de la realidad, representan formas de reescritura y, pues, de resemantización. Para Ester Newton, el camp representa una filosofía de la transformación de la falta de coherencia que pone en discusión la noción de normalidad. Cf. Sontag S., «Notes on Camp», Partisan Review, n 4, 1964, pp. 515-530; Newton, E., Mother Camp, cit. 
Devor, Holly (1999): FTM: Female-to-Male Transsexuals in Society, Indiana, Indiana University Press, 1999.

Douglas, Mary (1966, 2002): Purity and Danger. An Analysis of Concepts of Pollution and Taboo, Routledge, New York.

Ekins, Richard (1997): Male femaling. A grounded theory approach to cross-dressing and sex-changing, Routledge, New York.

Ekins, Richard y King, David (eds.) (1996): Blending genders. Social aspects of crossdressing and sex-changing, Routledge, New York.

Epstein, Julia y Straub, Kristina (eds.) (1991): Bodyguards: the cultural politics of gender ambiguity, Routledge, New York.

Foucault, Michel (1976): La volonté de savoir, Gallimard, Paris.

Foucault, Michel (1976): Historie de la sexualité, Gallimard, Paris.

Frazer, James (1915): The golden bough. A study in magic and religion, Macmillan, New York.

Garber, Marjorie (1992): Vested interests. Cross-dressing and cultural anxiety, Routledge, New York.

Hallett, Judith P. y Skinner, Marilyn B. (eds.) (1997): Roman sexualities, University Press, Princeton.

Hirschfeld, Magnus (1910): Die Transvestiten: Eine Untersuchung über den erotischen Verkleidungstrieb, mit umfangreichem kasuistischem und historischem Material, Verlag Alfred Pulvermacher, Berlin.

Newton, Esther (1972): Mother Camp. Female Impersonators in America, Chicago.

Penner, Todd y Vander Stichele, Caroline (2007): Mapping gender in ancient religious discourses, Brill, Leiden-Boston.

Storr, Anthony (1991): «The Psychopathology of Fetischism and Transvestitism», en Samuels, Andrew (eds.): Psychopathology: contemporary Jungian perspectives, The Guilford Press, New York, pp. 255-274.

Summers, Claude J. (eds.) (2004): The queer Encyclopedia of the visual arts, Cleis, San Francisco.

Von Humboldt, Wilhelm (1836): Über die Verschiedenheit des menschliches Sprachbaues, Akademie, Berlin.

Wheelwright, Julie (1989): Amazons and military maids: women who dressed as men in the pursuit of life, liberty and happiness, Pandora Press, London.

Zeitlin, Froma I., Winker, John J. \& Halperin, David M. (eds.) (1991): Before sexuality. The construction of erotic experience in the ancient Greek world, University Press, Princeton. 